\title{
Noninvasive Laboratory Markers as A Predictor of Esophageal Varices in Egyptian Cirrhotic Patients
}

\author{
A.A.Mostafa $^{1}$, H.S.Alegaily ${ }^{1}$, H.S.Mohammed ${ }^{2}$ and M.E.Abdelati ${ }^{2}$ \\ ${ }^{1}$ Hepatology Gastroenterology and Infection Diseases Dept., Faculty of Medicine, Benha Univ., Benha, Egypt \\ ${ }^{2}$ Kafr El Sheikh Liver Research Center, Kafr El Sheikh, Egypt \\ E-Mail:drmohammed6789@gmail.com
}

\begin{abstract}
Esophageal varices are one of the main complications of liver cirrhosis. Upper gastrointestinal endoscopy is the gold standard for the detection of esophageal varices. However, this is invasive and costly. The purpose of this study was to assess the predictive values of the noninvasive tests in detecting the presence of OV, so this study aimed to determine the role of Combined albumin-bilirubin grade and platelets (ALBI-PLT score), albumin-bilirubin score (ALBI) and Pugh (CP) Score as non -invasive markers in the prediction of esophageal varices and its grading in cirrhotic patients. This cross-sectional study was carried out at Hepatology, Gastroenterology and Infectious Diseases department, Benha University Hospital and at Kafr El- Shiekh Liver Research center, Egypt including 240 patients with liver cirrhosis randomly selected presenting for screening for esophageal varices. All subjects were subjected to full history taking, clinical examination and laboratory inv investigations including CBC, liver and renal function tests and Calculation of albumin-bilirubin score (ALBI), combined albumin-bilirubin grade and platelets (ALBI-PLT) and Pugh (CP) Score. Then Upper Gastrointestinal Endoscopy done by expert endoscopist to detect the presence or absence of esophageal varices, sites of varices, grades of varices. ALBI, ALBI-PLT, Child-Pugh (CP) Score are good predictors for presence of esophageal varices and its size . Combined albumin-bilirubin grade and platelets (ALBIPLT) could be used as a non-invasive predictor of presence of esophageal varices which may help in reducing unnecessary endoscopies .
\end{abstract}

\section{Introduction}

Portal hypertension is a common clinical syndrome, defined by a pathologic increase in the portal venous pressure. Increased resistance to portal blood flow, the primary factor in the pathophysiology of portal hypertension, is in part due to morphological changes occurring in chronic liver diseases. This results in re-routing of blood flow away from the liver through collateral pathways to decrease pressure in systemic veins[1]. The esophageal varices exist in $40 \%$ and $60 \%$ in compensated and de-compensate $\mathrm{d}$ disease during the diagnosis of liver cirrhosis and ascites [2].

The prevalence for esophageal varices (EVs) increases as $5 \%$ per year with $5-10 \%$ progression rate from small to large varices [3 ].

So, the Baveno consensus on portal hypertension in its first five editions had recommended surveillance with periodic upper endoscopies in these patients to identify in a timely fashion the development of esophageal varices and initiate a primary prophylactic strategy in those at a high risk of bleeding [4].

Liver cirrhosis is generally characterized by a long phase of compensated disease and the development of non-invasive methods for liver fibrosis assessment has meant that increasing numbers of patients are being diagnosed with cirrhosis at an early stage prior to the development of portal hypertension [5].

However, Fewer than $50 \%$ of cirrhotic patients have varices at screening endoscopy and most have small-sized varices with low risk of bleeding [6].

thus, a large proportion of cirrhotic patients do not present with high risk varies, thus making endoscopy a non-ideal screening test that is associated with significant costs and patient discomfort [7].
Noninvasive predictors of EVs represent a special importance in developing countries like Egypt where it is not easy to perform screening endoscopies to the huge number of patients with liver cirrhosis [8].

Noninvasiveness has become a major goal in hepatology in the latter years, since several serum markers and imaging methods have been demonstrated to correlate well with fibrosis stage and tend to replace liver biopsy, and there is a close relationship between liver fibrosis, portal hypertension and oesophageal varices, several of these methods have been tried for the noninvasive assessment of portal hypertension or presence of oesophageal varices [9].

Recently, the albumin-bilirubin (ALBI) score has been established as a more convenient and evidence-based model to assess the severity of liver dysfunction in patients with hepatocellular carcinoma (HCC) [10].

This study aimed to determine the role ALBI, combined ALBI-PLT and Child-Pugh (CP) Scores (as non-invasive markers) in the prediction of esophageal varices and discrimination between its grading in cirrhotic patients.

\section{Subjects and Methods 2.1 Subjects}

This cross sectional study was conducted on 240 cirrhotic patients (diagnosis of cirrhosis based on clinical, laboratory and radiological data) selected randomly presented for screening for esophageal varices at Hepatology, Gastroenterology and Infectious Diseases department, Benha University Hospital Hospitals and to Kafr El- Shiekh Liver Research center .Patients with age less than18 years old, Previous history of upper gastrointestinal bleeding, patients who underwent surgical intervention for portal hypertension, or transjugular intrahepatic 
portosystemic stent shunt placement were excluded from the study.

All patients gave informed written consents for participation in this study and this protocol was approved by the Medical Ethical Committee of Benha University according to the World Medical Association Declaration of Helsinki [11].

\subsection{Methods}

All Participants were subjected to:

1. Full history taking and clinical examination.

2. Laboratory investigations included:

3. Laboratory investigations:

4. Complete blood count (CBC).

5. -Liver function tests: liver enzymes: Aspartate amino transferase (AST) IU/L and Alanine amino transferase (ALT) IU/L, bilirubin: total \& $\operatorname{direct}(\mathrm{mg} / \mathrm{dl})$, albumin(gm/dl), Prothrombin time (sec), activity (\%) and international Neutralization Ratio (INR).

6. Kidney function tests: creatinine and urea.

7. Abdominal US: for evaluation of presence of cirrhosis, periportal fibrosis, diameter of the right lobe of the liver, spleen size, Ascites, focal lesions, portal vein diameter (PVD), portosystemic collaterals.

8. Calculation of albumin-bilirubin ratio (ALBI), combined albumin-bilirubin ratio and platelets (ALBI-PLT) and Child-Pugh (CP) Scores-Pugh (CP) Scores: The ALBI grade was calculated using the following equation: $0.66 \times$ $\log 10$ bilirubin level $-0.085 \times$ albumin level. The ALBI grades were stratified into grade $1(\leq-2.60)$, grade $2(-2.59$ to -1.39$)$, and grade $3(>-1.39)$.

9. The ALBI-PLT score was calculated by adding ALBI grade and points based on platelet count (1 point if platelet count $>150,000 / \mathrm{mm} 3$ and 2 points if $<150,000 / \mathrm{mm} 3) .12$

10. Pugh (CP) Score: The scoring method based on patient hepatic encephalopathy status, ascites assessment, INR, bilirubin and serum albumin values. Each measure is scored 1-3 and classified as: Class A: 5-6, Class B: 7-9, Class C: 10-15.13

11. Upper Gastrointestinal Endoscopy by expert endoscopist using (Olympus Q180 and Q240Japan) to detect the presence or absence of esophageal and gastric varices, sites of varices, grades of varices. At endoscopy, the esophageal varices were graded as large (Grade III-IV) or small (Grade I-II), based on Paquet's grading system:
Grade I: Microcapillaries located in distal esophagus or esophago-gastric junction.

Grade II: One or two small varices located in the distal esophagus.

Grade III: Medium-sized varices of any number.

Grade IV: Large-sized varices in any part of esophagus [14].

\subsection{Statistical analysis}

Data were analyzed using the statistical program for social sciences, SSPS version 26.0 (Chicago, USA). Quantitative data were expressed as mean \pm SD and qualitative data were expressed as frequency and percentage. Independent samples t-test of significance was used when comparing two means, Receiver operating characteristic (ROC) curve analysis was used to find the overall predictivity of parameters and find the best cutoff value for detection, along with sensitivity and specificity, the confidence interval was set to $95 \%$ and the margin of error accepted was set to $5 \%$. the Mann-Whitney U-test used to compare two nonparametric quantitative variables, $\mathrm{p}<0.05$ was considered statistically significant [14].

\section{Results}

This study included two hundred and forty cirrhotic patients with a mean age of $(57.40 \pm 17.40)$ years, they were $144(60 \%)$ male and 96 (40\%) females. Patient's characteristics, laboratory investigations and ultrasound examination are summarized in Table (1).

Regarding the presence of EV ALBI, ALBI-PLT, Child-Pugh (CP) Score are good predictors for presence of oesophageal varices. ALBI at cut-off >-2.6 can predict oesophageal varices with sensitivity 84.77 , specificity 53.06, PPV 82.4, NPV 45.6, AUC 0.711 and $\mathrm{P}$ value $<0.001$ Fig (1), ALBI- PLT at cut-off $>2$ can predict oesophageal varices with sensitivity 95.48 , specificity 87.76, PPV 95.9, NPV 97.7, AUC 0.982 and $\mathrm{P}$ value $<0.001$ Fig (2).

Regarding differentiation between Small OVs and large OVs, this study showed that ALBI, ALBIPLT, Child-Pugh (CP) Scores could be used in prediction of size of OVs, ALBI at cut-off >-2.03 can predict the size with sensitivity 91.28 , specificity 90.75, PPV 93.8, NPV 90.9, AUC 0.971 and P value $<0.001$. (Fig. 3) ALBI-Platelets at cut-off >3 can predict the size with sensitivity 59.48, specificity 93.44, PPV 96.6, NPV 53.8, AUC 0.864 and P value $<0.001$ Fig $(r)$.

Table (1) Patient's characteristics, laboratory investigations and ultrasound examination.

\begin{tabular}{lcc}
\hline Age $($ year $)$ & Range & $\mathbf{4 0}-\mathbf{7 4 . 4 0}$ \\
\cline { 2 - 3 } & Mean \pm SD & $\mathbf{5 7 . 4 0 \pm 1 7 . 4 0}$ \\
\hline Sex & Male & $144(60 \%)$ \\
& Female & $96(40 \%)$ \\
Medical diseases & DM & $60(25 \%)$
\end{tabular}


Table (1) Continue

\begin{tabular}{|c|c|c|}
\hline & $\mathrm{HTN}$ & $33(13.75 \%)$ \\
\hline \multirow[t]{5}{*}{ Cause of cirrhosis } & $\mathrm{HCV}$ & $205(85.42 \%)$ \\
\hline & $\mathrm{HBV}$ & $14(5.83 \%)$ \\
\hline & $\mathrm{BHF}$ & $47(19.58 \%)$ \\
\hline & AIH & $6(2.5 \%)$ \\
\hline & Others & $21(8.75 \%)$ \\
\hline \multirow[t]{3}{*}{ Child-Pugh (CP) Score } & A & $129(53.75 \%)$ \\
\hline & B & $72(30 \%)$ \\
\hline & $\mathrm{C}$ & $39(16.25 \%)$ \\
\hline Hepatic encephalopathy & 0 & $168(70 \%)$ \\
\hline \multirow[t]{2}{*}{ Grade } & $1-2$ & $51(21.25 \%)$ \\
\hline & $3-4$ & $6(2.5 \%)$ \\
\hline \multirow[t]{2}{*}{ Hb $(g m / d L)$} & Range & $5.6-17$ \\
\hline & Mean \pm SD & $9.99 \pm 1.99$ \\
\hline \multirow[t]{2}{*}{ Platelet $\left(* 10^{3} / \mathrm{mm}^{3}\right)$} & Range & $35-255$ \\
\hline & Mean \pm SD & $105.4 \pm 47.1$ \\
\hline \multirow[t]{2}{*}{ ALT (mg/dL) } & Range & $15-79$ \\
\hline & Mean \pm SD & $31.81 \pm 11.59$ \\
\hline \multirow[t]{2}{*}{ AST (mg/dL) } & Range & $18-128$ \\
\hline & Mean \pm SD & $38.86 \pm 17.76$ \\
\hline \multirow[t]{2}{*}{ Albumin (g/dL) } & Range & $1.7-4.6$ \\
\hline & Mean \pm SD & $3.3 \pm 0.71$ \\
\hline \multirow[t]{2}{*}{ Total bilirubin (mg/dL) } & Range & $0.5-4.6$ \\
\hline & Mean \pm SD & $1.5 \pm 0.85$ \\
\hline \multirow[t]{2}{*}{ Direct bilirubin (mg/dL) } & Range & $0.1-1.78$ \\
\hline & Mean \pm SD & $0.51 \pm 0.38$ \\
\hline \multirow[t]{2}{*}{ INR } & Range & $1.1-3.29$ \\
\hline & Mean \pm SD & $1.41 \pm 0.3$ \\
\hline \multirow[t]{2}{*}{ Creatinine (mg/dL) } & Range & $0.5-6$ \\
\hline & Mean \pm SD & $1.14 \pm 0.64$ \\
\hline Cirrhosis & $\mathrm{N}(\%)$ & $240(100 \%)$ \\
\hline Bilharzial hepatic fibrosis & $\mathrm{N}(\%)$ & $47(19.5 \%)$ \\
\hline \multirow[t]{2}{*}{ Spleen size $(\mathbf{c m})$} & Range & $11-21$ \\
\hline & Mean \pm SD & $16.63 \pm 2.3$ \\
\hline \multirow[t]{4}{*}{ Ascites } & No & $156(65 \%)$ \\
\hline & Mild & $63(26.25 \%)$ \\
\hline & Moderate & $18(7.5 \%)$ \\
\hline & Massive & $3(1.25 \%)$ \\
\hline \multirow[t]{2}{*}{ Portal vein diameter $(\mathrm{mm})$} & Range & $1.1-17$ \\
\hline & Mean \pm SD & $12.96 \pm 2.67$ \\
\hline Portal vein thrombosis & $\mathrm{N}(\%)$ & $21(8.75 \%)$ \\
\hline $\mathrm{HCC}$ & $\mathrm{N}(\%)$ & $23(9.58 \%)$ \\
\hline
\end{tabular}

As regard noninvasive predictors of esophageal varices are shown in table (2).

Table (2) Noninvasive predictors of esophageal varices.

\begin{tabular}{|c|c|c|}
\hline \multirow[t]{3}{*}{ Child-Pugh (CP) Score } & $\mathbf{A}$ & $129(53.75 \%)$ \\
\hline & $\mathbf{B}$ & $72(30 \%)$ \\
\hline & $\mathbf{C}$ & $39(16.25 \%)$ \\
\hline \multirow[t]{3}{*}{ ALBI } & Grade 1 & $67(27.9 \%)$ \\
\hline & Grade 2 & $100(41.7 \%)$ \\
\hline & Grade 3 & $73(30.4 \%)$ \\
\hline \multirow[t]{4}{*}{ ALBI - PLT } & 2 & $50(20.83 \%)$ \\
\hline & 3 & $30(12.5 \%)$ \\
\hline & 4 & $88(36.67 \%)$ \\
\hline & 5 & $72(30.0 \%)$ \\
\hline
\end{tabular}


Table (3) Diagnostic performance of ALBI, ALBI - PLT, Child-Pugh (CP) Scores-Pugh (CP) score in prediction of OV.

\begin{tabular}{lllllll}
\hline Cut-off & Sensitivity & Specificity & PPV & NPV & AUC & P value \\
\hline ALBI >-2.6 & 84.77 & 53.06 & 82.4 & 45.6 & 0.711 & $<0.001^{*}$ \\
ALBI-PLT $>$ 2 & 95.48 & 87.76 & 95.9 & 97.7 & 0.982 & $<0.001^{*}$ \\
$\begin{array}{l}\text { Scores-Pugh }(\text { CP) } \\
\text { Scores }>\text { 6 }\end{array}$ & 59.59 & 92.96 & 95.1 & 37.2 & 0.843 & $<0.001^{*}$ \\
\hline
\end{tabular}

*significant as $\mathrm{P}$ value $<0.05$

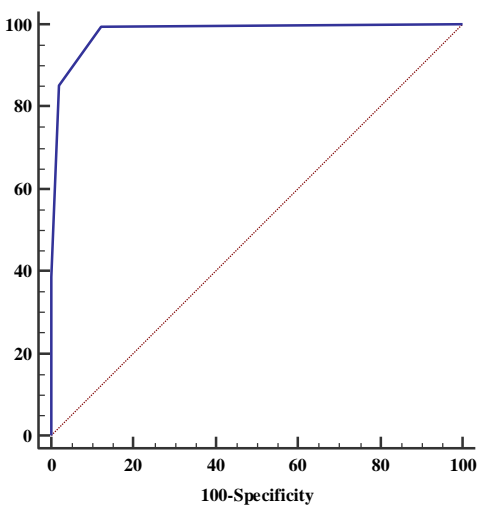

Fig (1) ROC curve of ALBI-platelets to diagnose OVs

Table (4) Diagnostic performance of ALBI, ALBI - PLT, Child-Pugh (CP) score in prediction of size of OVs.

\begin{tabular}{lllllll}
\hline Cut-off & Sensitivity & Specificity & PPV & NPV & AUC & P value \\
\hline ALBI $>-\mathbf{2 . 0 3}$ & 91.28 & 90.75 & 93.8 & 90.9 & 0.971 & $<0.001^{*}$ \\
ALBI-PLT $>\mathbf{3}$ & 59.48 & 93.44 & 96.6 & 53.8 & 0.864 & $<0.001^{*}$ \\
Child-Pugh (CP) & 84.89 & 92.62 & 90.5 & 71.6 & 0.920 & $<0.001^{*}$ \\
Scores $>$ 6 & & & & & & \\
\hline
\end{tabular}

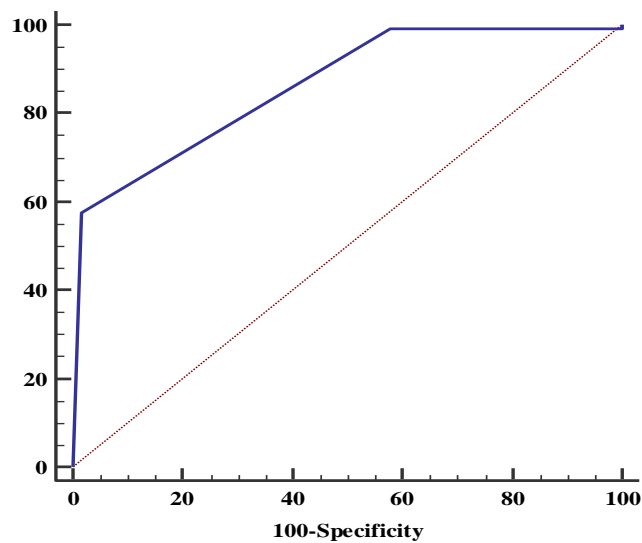

Fig (2) ROC curve of ALBI-PLT to predict the size.

\section{Discussion}

The current study assessed ALBI, ALBI-PLT and Child-Pugh (CP) Score as a noninvasive method for diagnosis and grading of esophageal varices in (240) patients with liver cirrhosis.

In the present study, platelet count was significantly lower in patients with esophageal varices than those without esophageal varices with $(\mathrm{p}<0.001)$, and was statistically significantly lower in patients with large varices compared to patients with small varices.

Our results came in agreement with Sarwar et al., (2005) also Sen and Griffiths (2008) who concluded that platelet count is a good marker to differentiate patients with or without OV [15 \&16].

In this study, there was a highly significant increase in the mean values of Child-Pugh score in cirrhotic patients with varices in comparison to other patients without varices. Also, there was a significant 
increase in the mean values of Child-Pugh score in cirrhotic patients with grade III and IV varices (large varices) in comparison to cirrhotic patients with grade I and II varices. and this is in agreement with Chang, et al., (2007) found the Child-Pugh score was one of the factors associated with the presence of large esophageal varices more so if the patient has two of these factors (alcohol consumption, splenomegaly and ascites), the sensitivity and negative predictive value for the presence of large esophageal varices and subsequently, increased risk of bleeding was $80 \%$ and $91.7 \%$ respectively [17].

However, many limitations of the $\mathrm{CP}$ score have been revealed for example, owing to the arbitrary use of cut-off points for continuous variables, a serum bilirubin level of $55 \mu \mathrm{mol} / 1$ has the same impact in the CP score as a level of $550 \mu \mathrm{mol} / 1$ [18].

As regard ALBI, we found that it was statistically significantly higher in patients with esophageal varices than those without esophageal varices $(p<0.001)$., and statistically significantly higher in patients with large oesophageal varices than those with small oesophageal varices $(\mathrm{p}<0.001)$.

These results agreed with the results of the study done by Gomaa et Al., 2018 in which they found that the diagnostic performance of ALBI in patients with $\mathrm{OV}$ and reported that that ALBI score could be used as a noninvasive predictor of esophageal varices with a cut-off value > - 2.2 with $96.7 \%$ sensitivity, $100 \%$ specificity, $100 \%$ PPV and $96.8 \%$ NPV, p-value < 0.001 . This difference may be due to change in number of patients as their study included smaller number of patients than our study (80 patients) [19].

$\mathrm{CP}$ and ALBI do not include factors that reflect portal hypertension such as the platelet count. They reflect liver synthetic dysfunction rather than portal hypertension.

In this study, ALBI-PLT was a good marker for prediction of oesophageal varices and for discrimination of large OV from small OV.

In our study, ALBI-PLT showed a higher accuracy in predicting oesophageal varices than the other tested variables as the study reported that $\mathrm{A}$ ALBI-PLT cut off value of $>2$ was highly predictive in the diagnosis of EV with a sensitivity of 95.48, specificity of 87.76 , PPV was 95.9, NPV was 97.7, AUC was 0.982 and $P$ value $<0.001$. For discrimination of large OV from small OV the ALBIPLT score showed significance and at cutoff value off $>3$ sensitivity was 59.48, specificity was 93.44, PPV was 96.6, NPV was 53.8, AUC was 0.864 and $\mathrm{P}$ value $<0.001$.

Chen et al., is the first to combine the use of ALBI grade and platelet count (ALBI-PLT score) to determine the risk of HRV in compensated patients with HCC, in the spirit of combining transient elastography and platelet count in the Baveno VI consensus. the ALBI-PLT model had excellent negative predictive value of HRV $(97.1 \%$ in the study cohort and $98.1 \%$ in the validation cohort, respectively) if patients had an ALBI-PLT score of 2 and concluded that the ALBI-PLT score could provide a simple, objective, accurate, and clinically applicable non-invasive tool to identify patients who are at a very low risk of HRV [12].

In addition to the excellent diagnostic value, ALBI-PLT has several clinical advantages. One can easily calculate ALBI-PLT at the bedside or in the outpatient clinic as it does not require standardization and is free of intra- and interobserver variability.

\section{Conclusion}

Combined ALBI grade and platelet count (ALBIPLT score) could be used as a serum non-invasive marker of presence of oesophageal varices which may help in reducing unnecessary endoscopies and good selection of patients who need endoscopy to decrease intervention and burden on endoscopy units.

\section{References}

[1] J.Bosch, R.J.Groszmann, and V.H. Shah ,Evolution in the understanding of the pathophysiological basis of portal hypertension: how changes in paradigm are leading to successful new treatments. J Hepatol, Vol.62(1), PP.S121-S130, 2015.

[2] A.Sarangapani, A.Shanmugam, M.Kalyanasundaram, B.Rangachari, P.Thangavelu, J.K.Subbarayan, Noninvasive prediction of large esophageal varices in chronic liver disease patients. Saudi J Gastroentero , Vol.16(1), PP.38-42, 2010.

[3] F.Barrera, A.Riquelme, A.Soza, A.Contreras, G.Barrios, O.Padilla, Platelet count/spleen diameter ratio for non-invasive prediction of high risk esophageal varices in cirrhotic patients. Ann Hepato, Vol.8(4), PP.325-30, 2009.

[4] R. De Franchis, Baveno VIF. Expanding consensus in portal hypertension: Report of the Baveno VI Consensus Workshop: Stratifying risk and individualizing care for portal hypertension. J Hepatol, Vol.63, PP.743-752, 2015.

[5] A.Berzigotti, S.Seijo, U.Arena, (): Elastography, spleen size, and platelet count identify portal hypertension in patients with compensated cirrhosis. Gastroenterology, Vol.144, PP.102-111, 2013.

[6] A.Binţinţan, I.Chira, and A.Mircea (): Ultrasoundbased diagnosis and staging of esophageal varices in liver cirrhosis. A systematic Non-invasive review of the literature published in the third millenium. Med Ultrason, , Vol.15(2), PP.116-24, 2013.

[7] A.Berzigotti, J.Bosch, T.D. Boyer, Use of noninvasive markers of portal hypertension and timing of screening endoscopy for gastroesophageal varices in patients with chronic liver disease. Hepatology, Vol.59, PP.729-731, 2014. 
[8] S.Esmat, D.Omran, Study of noninvasive predictors of portal hypertension in liver cirrhotic Egyptian patients. J Am Sci, Vol.7, PP.962-968, 2011.

[9] D.H.Leung, M.J.Finegold and B.L.Shneider , Diagnostic methods of cirrhosis and portal hypertension: Specifics of the pediatric population. In: Diagnostic Methods for Cirrhosis and Portal Hypertension. Springer, Vol.25, pp325-341, 2018.

[10]H.Zou, Y.Wen, K.Yuan, X. Y.Miao, L.Xiong, Combining albumin-bilirubin score with future liver remnant predicts post-hepatectomy liver failure in $\mathrm{HBV}$-associated $\mathrm{HCC}$ patients. Liver International, Vol.38(3) ,PP. 494-502, 2018.

[11]J.E. Idänpään-Heikkilä,Ethical principles for the guidance of physicians in medical research: the Declaration of Helsinki. Bull World Health Organ, Vol. 79(4), PP. 279-279, 2001.

[12]P.Chen, W.Hsieh, C.Su, M.Hou, Y.Wang , (): Combination of albumin-bilirubin grade and platelets to predict a compensated patient with hepatocellular carcinoma who does not require endoscopic screening for esophageal varices. Gastrointest Endosc , Vol.88, PP. 230-9, 2018.
[13] R. Pugh, I. Murray-Lyon, J. Dawson, Transection of the oesophagus for bleeding oesophageal varices. Br. J. Surg., vol, 60, PP. 646-649, 1973.

[14] K.J.Paquet:, Prophylactic endoscopic sclerosing treatment of the esophageal wall in varices: a prospective controlled randomized trial. Endoscopy, Vol. 14,PP. 4-5,1982.

[15] S.Sarwar, A.Khan, A.Alam ,Non-endoscopic prediction of presence of varices in cirrhosis. J Coll physicians Surg Pack. March; 15(9):528-31, 2005.

[16]S.Sen, J.Griffiths , Non-invasive prediction of oesophageal varices in cirrhosis. World J Gastroenterol 14:2454-5, 2008.

[17] M.H.Chang, J.H.Sohn, T.Y. Kim , Nonendoscopic predictors of large esophageal varices in patients with liver cirrhosis. Koream J. Gastroenterology, Vol.49 (6), PP.376-83, 2007.

[18]F.Durand, D.Valla, Assessment of the prognosis of cirrhosis: Child-Pugh versus MELD. J Hepatol, Vol. 42(1), PP.100-107,2005.

[19] A.Gomaa, S.Mohammed, W.Mousa, N.Hasan, M.Mhdy Evaluation of ALBI, MELD and ChildPugh (CP) Scores-Pugh Scores as non-Invasive Predictors of Esophageal Varices. Egy J Hosp Med , Vol.73 (8) ,PP.7358-7364, 2018. 